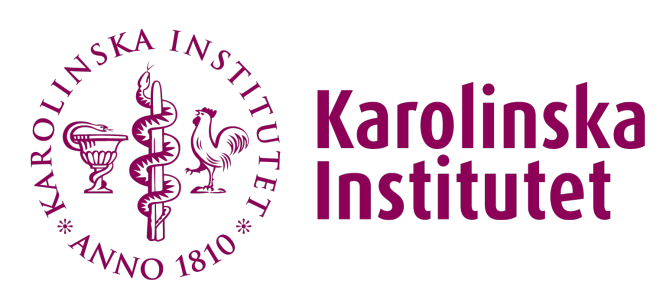

Karolinska Institutet

http://openarchive.ki.se

This is a Peer Reviewed Accepted version of the following article, accepted for publication in Epidemiology.

2013-04-12

\title{
Abdominal fat and male excess of esophageal adenocarcinoma
}

Lagergren, Katarina; Mattsson, Fredrik; Lagergren, Jesper

Epidemiology. 2013 May;24(3):465-6.

http://doi.org/10.1097/EDE.0b013e31828b0a26

http://hdl.handle.net/10616/41498

If not otherwise stated by the Publisher's Terms and conditions, the manuscript is deposited under the terms of the Creative Commons Attribution-NonCommercial-NoDerivatives License (http://creativecommons.org/licenses/by-nc-nd/4.0/), which permits non-commercial re-use, distribution, and reproduction in any medium, provided the original work is properly cited, and is not altered, transformed, or built upon in any way. 


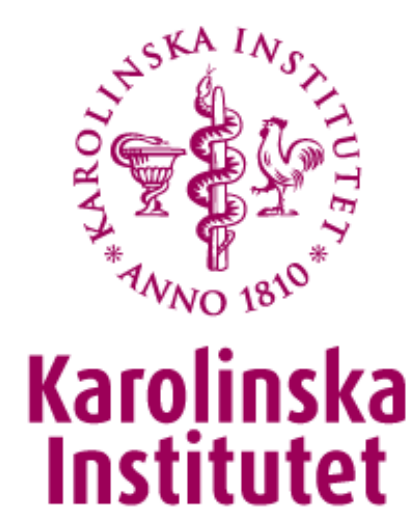

This is an author produced version of a paper published in Epidemiology. This paper has been peer-reviewed but does not include the final publisher proof-corrections or journal pagination.

Citation for the published paper:

Epidemiology. 2013 May;24(3):465-6.

Abdominal fat and male excess of esophageal adenocarcinoma

Lagergren, Katarina; Mattsson, Fredrik; Lagergren, Jesper

\section{DOI: 10.1097/EDE.0b013e31828b0a26}

Access to the published version may require subscription. Published with permission from: LWW 


\section{Abdominal fat and male excess of esophageal adenocarcinoma}

Authors: Katarina Lagergren ${ }^{1}$, Fredrik Mattsson ${ }^{1}$, Jesper Lagergren ${ }^{1,2}$.

Affiliations: ${ }^{1}$ Upper Gastrointestinal Research, Department of Molecular Medicine and Surgery, Karolinska Institutet, Stockholm, Sweden.

${ }^{2}$ Division of Cancer Studies, King's College London, United Kingdom.

Corresponding author: Jesper Lagergren

Address: Upper Gastrointestinal Research, Department of Molecular Medicine and Surgery, Karolinska Institutet, Norra Stationsgatan 67, level 2, 17176 Stockholm, Sweden.

E-mail: jesper.lagergren@ki.se, Telephone: + 468517760 12, Fax: + 46851776280

Keywords: Neoplasm; esophagus; obesity; sex ratio; central adiposity.

Financial support: The Swedish Research Council and the Swedish Cancer Society. The funding sources had no involvement in the study.

Conflict of interest statement: None declared.

Word count: 597

Total number of tables: 1

Total number of figures: $\mathbf{0}$

Number of references: 8 


\section{Introduction}

Esophageal and junctional adenocarcinoma (EAC) has a 7-to-1 male predominance.(1) The main risk factors for EAC, obesity and gastroesophageal reflux, are however equally distributed between sexes.(2-4) A predominantly abdominal fat distribution, typical for males, is however a stronger risk factor than $\mathrm{BMI},(5-8)$ and abdominal adiposity might facilitate reflux through increased intra-abdominal pressure. Abdominal adiposity has therefore been suggested to explain the male predominance of EAC. If true, the male-tofemale sex ratio in EAC would be low, or absent, among lean patients and high among overweight. We aimed to test this hypothesis. 


\section{Methods}

We analyzed data from a population-based nationwide Swedish case-control study, which has been described in detail elsewhere.(3) In brief, participants were prospectively included in 1995-1997. Cases were recruited from all 195 Swedish hospital departments involved in these patients. Tumor classification was comprehensive and uniform. Controls were randomly selected and frequency-matched for age and sex of EAC cases. Due to the matching on sex, controls were used to estimate person-years by employing data from the Swedish Register of the Total Population. At personal interviews information on BMI was assessed as: 1) 20 years before interview, 2) 20 years of age, 3) maximum adult BMI, and 4) minimum adult BMI. Incidence rate ratio estimated relative risk (RR). Person-years were calculated by age $(<60,60-69$, or $70-79)$, sex and $\mathrm{BMI}(<22,22-<25$, or $\geq 25)$ derived from the Swedish population. The BMI-distribution in controls was used to estimate person-years in the population. Poisson regression was used to calculate RR and $95 \%$ confidence intervals (CI), where log-transformed person-years were included in the model as an offset. To evaluate effect modification of BMI on the association between sex and EAC, we included age, sex, BMI and an interaction term between sex and BMI in the model. Likelihood ratio $\chi^{2}$ statistics was used to obtain $P$-value for test of effect modification. 


\section{Results}

Included were 451 EAC cases (85\% participation) and 820 controls (73\%). The male-tofemale ratio was 7-to-1. Half of the EAC cases were overweight (BMI $\geq 25) 20$ years before interview. There was no increased RR with increasing levels of BMI in any of the BMIassessments (Table). Regarding BMI 20 years before interview, the male predominance in overweight participants (RR $5.8,95 \% \mathrm{Cl} 3.9-8.8$ ) was not higher than in lean (RR 7.4, 95\% Cl 3.9-14.1). The corresponding RRs at age 20 were similar ( $R R 7.2,95 \% \mathrm{Cl} 3.6-14.3$ and RR 7.2, $95 \% \mathrm{Cl} 4.8-10.6$, respectively). Regarding minimum $\mathrm{BMI}$, there was rather a higher male predominance in lean participants, and for maximum BMI the male predominance was at least as strong in lean (Table). 


\section{Discussion}

This study provides no support for the hypothesis that an increased male predominance of EAC correlates with higher BMI.

The population-based design with high participation rates and the possibility to estimate person-years from population-based controls were prerequisites for the study. Recording of all Swedish residents enabled assessment of person-years at risk from which the cases were derived, and thus possible to adjust for age. Other advantages include the thorough tumor classification, personal interviews, and assessment of BMI at different time points. Among weaknesses is possible misclassification of BMI, but any such bias should be similar between cases and controls. Moreover, the low incidence of EAC in women reduced the statistical power.

In conclusion, although abdominal adiposity is clearly overrepresented in men and there is a strong male predominance in EAC, this population-based study with a nearly complete assessment of EAC cases in Sweden found no evidence of an increased age-adjusted male predominance with higher levels of BMI, which argues against abdominal obesity being a key factor in explaining the male predominance. 


\section{References}

1. Edgren G, Adami HO, Weiderpass Vainio E, Nyren O. A global assessment of the oesophageal adenocarcinoma epidemic. Gut. 2012. Epub 2012/08/25.

2. Hoyo C, Cook MB, Kamangar F, Freedman ND, Whiteman DC, Bernstein L, et al. Body mass index in relation to oesophageal and oesophagogastric junction adenocarcinomas: a pooled analysis from the International BEACON Consortium. International journal of epidemiology. 2012. Epub 2012/11/14.

3. Lagergren J, Bergstrom R, Lindgren A, Nyren O. Symptomatic gastroesophageal reflux as a risk factor for esophageal adenocarcinoma. The New England journal of medicine. 1999;340(11):825-31.

4. Lofdahl HE, Lu Y, Lagergren J. Sex-specific risk factor profile in oesophageal adenocarcinoma. British journal of cancer. 2008;99(9):1506-10.

5. Steffen A, Schulze MB, Pischon T, Dietrich T, Molina E, Chirlaque MD, et al. Anthropometry and esophageal cancer risk in the European prospective investigation into cancer and nutrition. Cancer Epidemiol Biomarkers Prev. 2009;18(7):2079-89.

6. MacInnis RJ, English DR, Hopper JL, Giles GG. Body size and composition and the risk of gastric and oesophageal adenocarcinoma. International journal of cancer. 2006;118(10):2628-31.

7. Corley DA, Kubo A, Zhao W. Abdominal obesity and the risk of esophageal and gastric cardia carcinomas. Cancer Epidemiol Biomarkers Prev. 2008;17(2):352-8.

8. Beddy P, Howard J, McMahon C, Knox M, de Blacam C, Ravi N, et al. Association of visceral adiposity with oesophageal and junctional adenocarcinomas. The British journal of surgery. 2010;97(7):1028-34. 
Table. Age-adjusted relative risk (RR) and 95\% confidence interval (CI) of esophageal or gastroesophageal junctional adenocarcinoma in categories of body mass index (BMI) in males compared to females (reference category).

\begin{tabular}{lllll}
\hline \multicolumn{5}{c}{ Body mass index } \\
BMI categories & $\begin{array}{l}\text { 20 years before } \\
\text { interview }\end{array}$ & $\mathbf{2 0}$ years of age & $\begin{array}{l}\text { Minimum as } \\
\text { adult }\end{array}$ & $\begin{array}{l}\text { Maximum as } \\
\text { adult }\end{array}$ \\
\hline & $\mathbf{R R}(\mathbf{9 5 \%} \mathbf{~ C l})$ & RR $(\mathbf{9 5 \%} \mathbf{C l})$ & RR (95\% Cl) & RR (95\% CI) \\
\cline { 2 - 5 }$<22$ & $7.4(3.9-14.1)$ & $7.2(4.8-10.6)$ & $7.9(5.4-11.5)$ & $7.5(1.7-33.8)$ \\
$22-<25$ & $5.3(3.5-8.1)$ & $4.2(2.7-6.5)$ & $3.6(2.4-5.5)$ & $8.5(4.5-15.9)$ \\
$\geq 25$ & $5.8(3.9-8.8)$ & $7.2(3.6-14.3)$ & $4.2(1.9-9.3)$ & $5.9(4.3-7.9)$ \\
$P$-value* & 0.68 & 0.17 & 0.03 & 0.55 \\
\hline
\end{tabular}

* Test of effect modification 